\title{
High resolution neutron radiography with very compact and efficient neutron collimators
}

\author{
A.S. Tremsin, ${ }^{a, 1}$ J.B. McPhate, ${ }^{a}$ E.H. Lehmann, ${ }^{b}$ J.V. Vallerga,,${ }^{a}$ O.H.W. Siegmund ${ }^{a}$ \\ and W.B. Feller ${ }^{c}$ \\ ${ }^{a}$ Space Sciences Laboratory, University of California at Berkeley, \\ Berkeley, CA 94720, U.S.A. \\ ${ }^{b}$ Paul Scherer Institut, Spallation Neutron Source Division, \\ CH-5232 Villigen, Switzerland \\ ${ }^{c}$ NOVA Scientific, Inc., \\ 10 Picker Rd., Sturbridge, MA 01566, U.S.A.
}

E-mail: ast@ssl.berkeley.edu

ABSTRACT: The spatial resolution in neutron imaging is often limited by the neutron beam divergence, especially in experiments where the distance between the sample and the detector cannot be very small. Imaging of relatively large or extended objects or tomographic experiments, where samples have to be rotated, typically require several to tens of centimeters distance between the sample and the detector. Moreover, some neutron imaging techniques require placement of components between the sample and the detector, as in the case of magnetic field imaging with polarized neutrons. Compact and efficient policapillary collimators can be an attractive alternative to the conventionally used beam limiting aperture approach. The possible improvement of spatial resolution by a policapillary collimator is studied in experiments where beam divergence limits the quality of neutron transmission imaging. A $\sim 7 \mathrm{~mm}$ thick collimator with $\sim 6 \mu \mathrm{m}$ capillaries demonstrates the possibility to decrease the neutron beam divergence and to diminish the image blurring. The improvement of out-of-angle neutron rejection and peak transmission of our future collimators will be crucial for their effective application in neutron imaging experiments.

KEYWORDS: Instrumentation for neutron sources; Neutron detectors (cold, thermal, fast neutrons); Neutron radiography

\footnotetext{
${ }^{1}$ Corresponding author.
} 


\section{Contents}

1 Introduction 1

2 Experimental setup $\quad 2$

3 Results and discussion $\quad 3$

3.1 Varied distance between the detector and the sample 4

3.2 Varied aperture 5

$\begin{array}{lll}3.3 & \text { Spatial resolution tests with a PSI test pattern } & 6\end{array}$

$\begin{array}{lll}3.4 & \text { Neutron refraction effect } & 7\end{array}$

4 Conclusions $\quad 8$

\section{Introduction}

The spatial resolution of thermal and cold neutron radiography and tomography is often limited by the divergence of the neutron beam. In the case of large or extended objects or when objects have to be rotated for tomographic imaging $[1,2]$ the distance between the sample and the detector active area cannot be set smaller than a particular value, typically ranging between few to tens of centimetres. At the same time, imaging of magnetic fields with polarized neutrons requires placement of an analyzer between [3] object and the detector, thus limiting spatial resolution even for a thin sample. The divergence of neutron beam in that case is very often the limiting factor for spatial resolution of neutron imaging. The widely used technique of beam collimation using an input aperture results in a compromise between neutron flux and the attainable spatial resolution due to the beam divergence. High spatial resolution imaging requires a high flux in within each pixel area, but reduction of the input aperture (intended for the improvement of image sharpness) lowers the flux available. Untill very recently, no efficient neutron optics existed which can shape the neutron beam into a collimated non-diverging uniform beam without significant losses of neutron intensity or introduction of strong image distortions below few hundred micrometer scales. The widely used Soller type collimators, consisting of many thin absorbing plates (e.g. silicon wafers coated with Gd-films [4]) operate in one dimension and typically have image distortions due to $>100 \mu \mathrm{m}$ internal structure [5]. Two 1-D collimators placed in sequence in the beam can provide 2-dimensional collimation in that configuration. However, stacking of such collimators requires relatively large distances $(\sim 40 \mathrm{~cm})$ and they cannot be placed in some existing neutron imaging facilities. In addition to that, in some cases the limited length of neutron flight path between the aperture and the detector does not allow high degree of collimation with a reasonable size apertures still providing sufficient neutron flux.

To fully utilize the recent progress in detection systems enabling spatial resolution on a sub- 15 $\mu \mathrm{m}$ scale [6]-[8] both high collimation and high intensity beams are required if reasonable image acquisition times are to be realized. Very compact (few mm thick) and efficient (as narrow as \pm 0.05 
degree) neutron polycapillary collimators $[9,10]$ can be a very attractive alternative to the single input aperture or Soller type collimator approaches and in some cases can substantially increase the neutron flux for a given beam divergence. It is achieved by beam collimation by millions of highly parallel pores with diameters on the scale of $<10 \mu \mathrm{m}$, which match the achievable detector spatial resolution, and thus do not introduce image distortions $[9,10]$.

We present the results of our experimental evaluation of a $\sim 7 \mathrm{~mm}$ thick polycapillary glass collimator with $6 \mu \mathrm{m}$ pores performed at a cold neutron facility FunSpin [11] at Paul Scherrer Institute. With the help of neutron collimator developed in collaboration between University of California at Berkeley and Nova Scientific we have demonstrated the potential for high resolution imaging on a beamline without a high degree of collimation by an aperture. The spatial resolution of images recorded close to the neutron waveguide output can be substantially improved with an easily insertable micropore collimator. These collimators can find their application not only in high resolution radiography and microtomography setups, but also be used in high resolution mapping of strain through neutron transmission diffraction [12], visualization of magnetic fields through neutron spin interaction [3] and many other applications, especially where the distance from the sample to the detector cannot be reduced - as in the case where a neutron polarizer is required between the detector and the sample. Also those collimators can be used for scatter rejection when they are placed between the object and the detector, substantially increasing the accuracy of quantitative neutron transmission measurements [13].

\section{Experimental setup}

The experiments reported in this paper were performed at a cold neutron beamline facility FunSpin at Paul Scherrer Institute [11] which was not configured for a high resolution neutron radiography at that time. An interchangeable aperture of $1 \mathrm{~cm}, 2 \mathrm{~cm}$ and $4 \mathrm{~cm}$ diameter was placed in the beam at the exit of the neutron waveguide and a relatively high resolution neutron counting detector [14] was installed at a $116 \mathrm{~cm}$ distance from the aperture. The divergence of the neutron beam in that configuration was respectively with $\mathrm{L} / \mathrm{D}=116,58$ and 29 . A compact polycapillary collimator with $\sim 7 \mathrm{~mm}$ thickness doped with Boron atoms was installed between the aperture and the detector in some measurements (figure 1) and removed during others to compare the achievable resolution in different experimental configurations. The distance between the sample and the detector was varied between 1 and $8 \mathrm{~cm}$. The measured neutron flux at the detector input with different apertures and the collimator in the beam is summarized in table 1 . The neutron flux was reduced by the polycapillary collimator by a factor of $\sim 20$ for all apertures. The reduction of flux is attributed to two factors. First of all, out-of-angle neutrons were rejected by the collimator, and secondly, the collimator itself was not an optimized device for the best peak transmission yet. We have measured the transmission of this collimator as a function of rocking angle (figure 2) at a PSI thermal neutron beamline facility NEUTRA. The $25 \%$ peak transmission will be improved in the next generation device to a 50-65 \% level. Another deficiency of this particular collimator is $~ 3 \%$ transmission for out-of-angle neutrons, which will also be reduced to $<0.1 \%$ levels by proper doping of internal structures. It should be noted here, that $3 \%$ transmission is measured for the integrated thermal neutron spectrum, while the rejection of cold neutrons is much better due to higher cross section of neutron absorption reaction inside the collimator. 

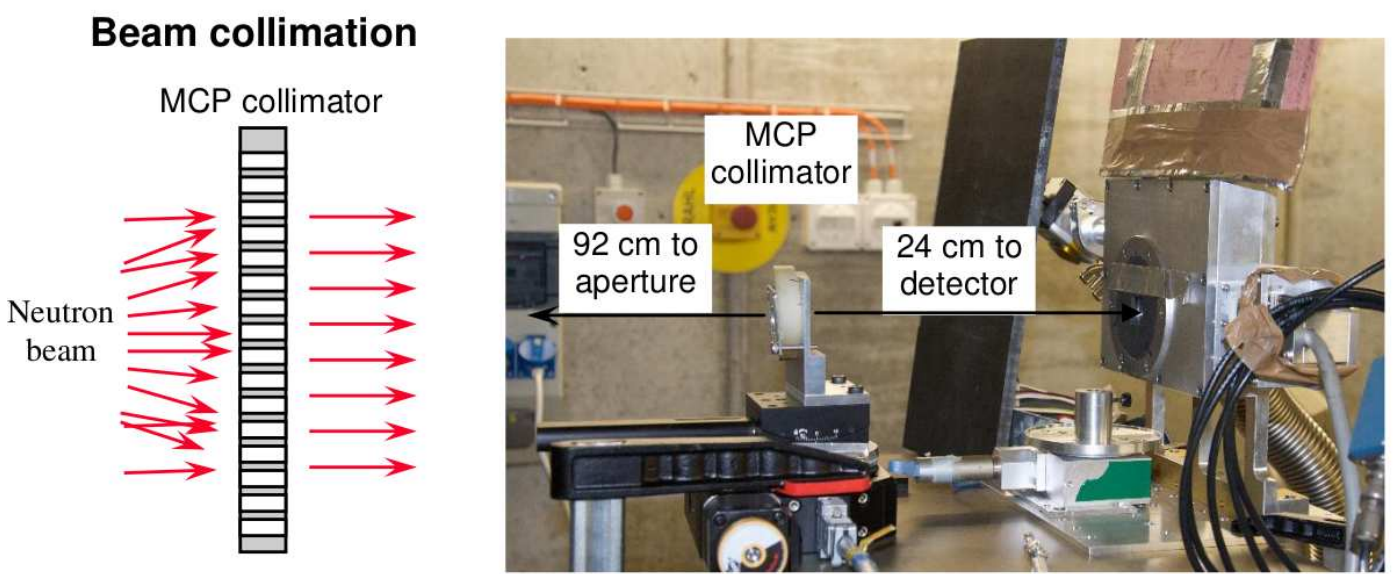

Figure 1. Beam collimation with MCP. Experimental setup at the FunSpin facility at Paul Scherrer Institute. The MCP collimator with L/D 1000:1 and $\sim 6 \mu$ m unetched pores is installed in the beam at $\sim 92 \mathrm{~cm}$ distance from the beam aperture. Collimator contained $\sim 22000$ micropores per $\mathrm{mm}^{2}$ area. Detector is positioned $\sim 24 \mathrm{~cm}$ from the collimator. Samples are mounted between the collimator and the detector. Various apertures (with diameter of $1 \mathrm{~cm}, 2 \mathrm{~cm}$ and $4 \mathrm{~cm}$ ) defining neutron beam divergence were used in the measurements. Beam divergences at these apertures are $\mathrm{L} / \mathrm{D}=116$ for a $1 \mathrm{~cm}$ aperture, $\mathrm{L} / \mathrm{D}=58$ for a 2 $\mathrm{cm}$ aperture and $\mathrm{L} / \mathrm{D}=29$ for a $4 \mathrm{~cm}$ aperture.

Table 1. Neutron beam fluxes at the cold neutron FunSpin facility measured with different input apertures.

\begin{tabular}{|l|l|l|}
\hline Aperture diameter & Flux $\mathbf{n} / \mathbf{c m}^{2} / \mathbf{s , n o}$ collimator & Flux $\mathbf{~} / \mathbf{c m}^{2} / \mathbf{s}$, with collimator \\
\hline $1 \mathrm{~cm}$ & $1 \mathrm{e} 7$ & $5.8 \mathrm{e} 5$ \\
\hline $2 \mathrm{~cm}$ & $3.2 \mathrm{e} 7$ & $1.2 \mathrm{e} 6$ \\
\hline $4 \mathrm{~cm}$ & $4.5 \mathrm{e} 7$ & $1.7 \mathrm{e} 6$ \\
\hline
\end{tabular}

\section{Results and discussion}

A set of neutron transmission radiographic images was taken with several test samples. One of them consisted of several small objects of different materials shown in figure 3 . The first tests were performed at a very short distance between the detector active area and the sample to establish the baseline of achievable spatial resolution. Obviously, at a short distance to the active area the beam divergence does not substantially blur the detected image and therefore we should not expect an improvement of the spatial resolution by a neutron collimator. However, it is important to show that the presence of our compact collimator does not introduce image distortions, as it was predicted by our modelling $[9,10]$. Figure 4 shows the transmission radiographies of the test mask obtained with the collimator in the beam and without it. The integration time was adjusted to acquire approximately same neutron statistics for both configurations. No image distortions by a collimator were found in this experiment. Moreover, some improvement of spatial resolution was observed as indicated by cross sections through the images shown in figure 4 . 


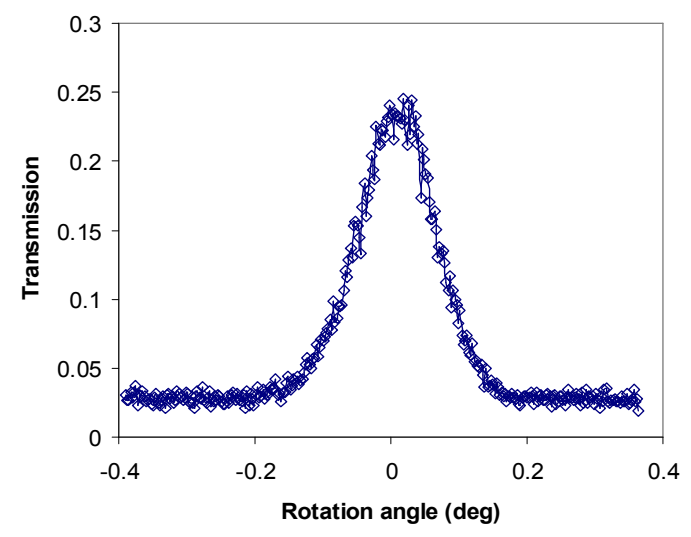

Figure 2. The thermal neutron transmission of the collimator used in the experiment as a function of rotation angle (rocking curve). Unetched pores limit the peak transmission to only $25 \%$. Presently it is very difficult to manufacture a single unit etched collimator with $\mathrm{L} / \mathrm{D} ; 250$ and good pore alignment due to the limited depth of the chemical etch. the alignment of pores along the pore axis is better than 0.05 degree.

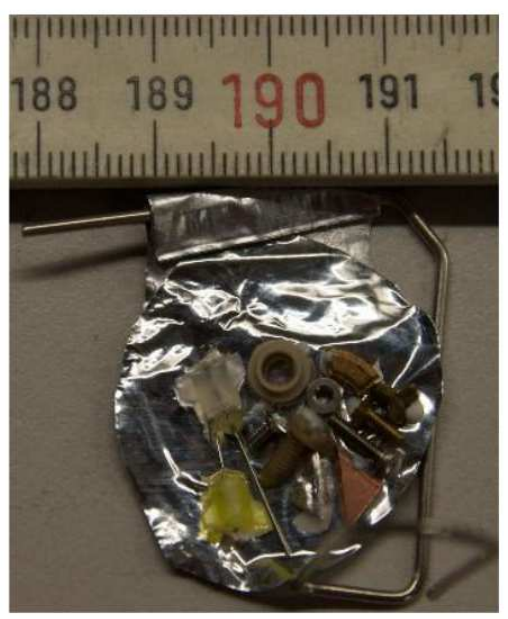

Figure 3. Imaging mask containing $\mathrm{M} 2$ steel and plastic screws, various pieces of copper, $\mathrm{Mg}$ and plastic used for the resolution tests shown in figure 4 . The objects are glued to an aluminium foil.

\subsection{Varied distance between the detector and the sample}

Another test object - a small wrist watch (figure 5) was installed at $1 \mathrm{~cm}$ and $8 \mathrm{~cm}$ distances from the detector active area to investigate the possible improvement of spatial resolution by the policapillary collimator. In case of $1 \mathrm{~cm}$ distance the improvement of spatial resolution is very marginal, as in the previous measurement shown in figure 4 . However, at $8 \mathrm{~cm}$ distance to the active area, the image blurring severely degrades the spatial resolution on both images (with collimator and without it), although the spatial resolution achieved with the collimator is substantially improved compared to the non-collimated setup. The smallest $1 \mathrm{~cm}$ aperture was used in these measurements. A much longer integration was needed to achieve the same statistics. Therefore the improved peak 

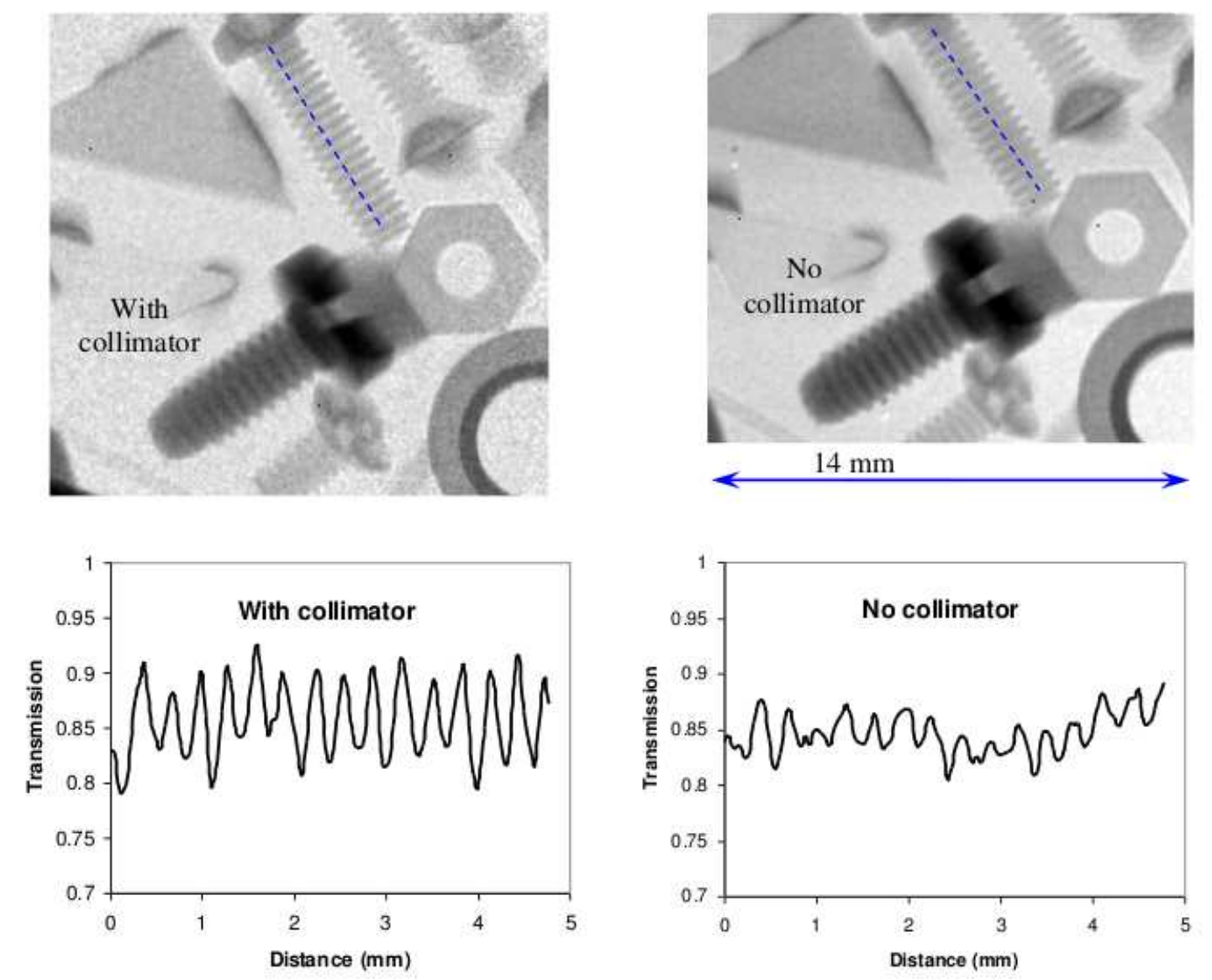

Figure 4. Transmission neutron radiography of test object shown in Fig. 3. The object was installed at $\sim 10$ $\mathrm{mm}$ distance to active area of the detector. $1 \mathrm{~cm}$ beam aperture is used. Image integration time $\sim 10$ minutes (with collimator) and 1 minute (without collimator). The threads on the M2 steel screw are better resolved at the image obtained with the collimator which improved the beam divergence. However, reduction of beam flux by the collimator required a longer integration time. Also the intensity of neutron beam at the edges of the image with the collimator is lower than in the centre as the collimator produces the image of the aperture at the detector plane. Both images are normalized by the corresponding open beam images.

transmission of our next generation collimator will be very important and will substantially reduce the required integration times. The blurring would be stronger with larger apertures, as will be shown later in section 3.2.

\subsection{Varied aperture}

In the next measurement we have fixed the distance to the detector to $42 \mathrm{~mm}$ and varied input aperture, figure 6 . The beam collimation with a policapillary device improved spatial resolution for both 1 and $2 \mathrm{~cm}$ apertures, as expected. However, the resolution of the collimated configuration also degraded between 1 and $2 \mathrm{~cm}$ apertures, which in ideal case should not happen. This degradation is due to the $\sim 3 \%$ transmission of this particular collimator for out-of-angle neutrons, which results 


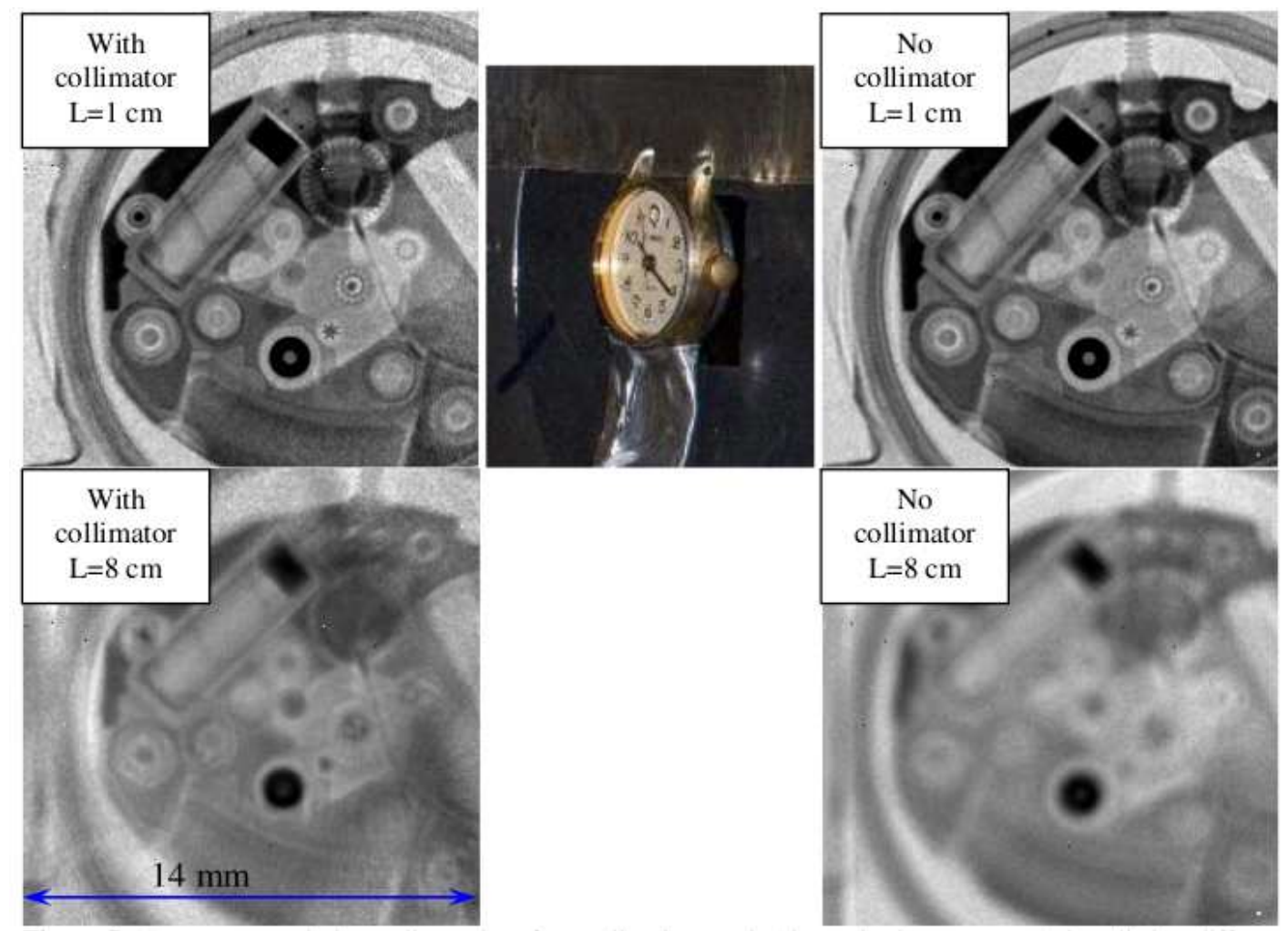

Figure 5. Neutron transmission radiography of a small wrist watch (shown in the upper row), installed at different distances to the detector active area, noted as $\mathrm{L}$ in image inserts. $1 \mathrm{~cm}$ aperture used in the measurements. Image acquisition $\sim 10$ minutes and $33 \mathrm{sec}$ for the collimator installed and no collimator measurements, respectively. At $\mathrm{L}=1 \mathrm{~cm}$ the beam divergence does not reduce the resolution of radiography. The additional beam shaping with the collimator does not improve the image quality at the $\sim 55 \mu$ m detector resolution level. However, at a large distance to the active area the resolution of the image is substantially improved by the collimator. The distance of $8 \mathrm{~cm}$ to the active area is not unreasonably large as in some experiments components have to be installed between the object and the detector, as in case of imaging with polarized neutrons - an analyzer is installed behind the object.

in the increased flux of uncollimated neutrons when beam aperture is increased. We expect that our future device with a better rejection ratio will not exhibit that degradation.

\subsection{Spatial resolution tests with a PSI test pattern}

A more detailed measurements on the achievable spatial resolution were performed with a PSI test pattern, produced by a deposition of Gd thin layer on a Si wafer [15], figures 7, 8. Both distance to the detector and the input aperture were changed in these experiments. A substantial improvement of spatial resolution was observed with a policapillary collimator. At $1 \mathrm{~cm}$ distance very little image degradation was observed with collimator, while the non-collimated configuration exhibited a substantial blurring at the $4 \mathrm{~cm}$ aperture. Despite the fact that almost the same spatial resolution was achieved with a $30 \mathrm{~s}$ integration $(1 \mathrm{~cm}$ aperture with no collimator and $4 \mathrm{~cm}$ aperture with collimator), these results indicate that the improved out-of-angle rejection of our future collimator 

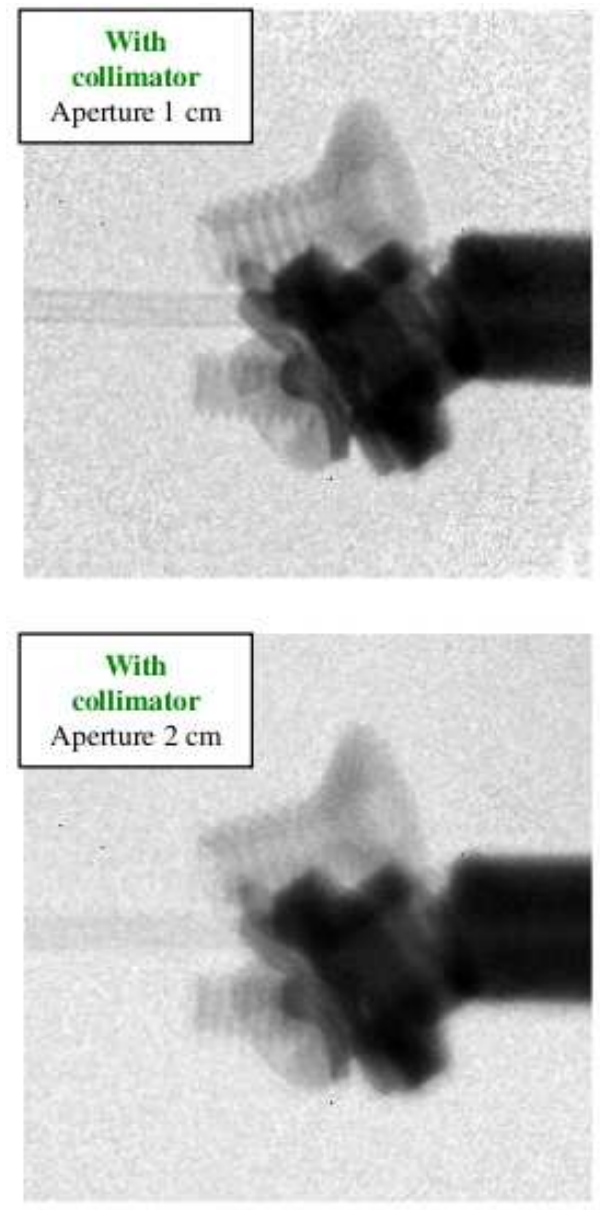
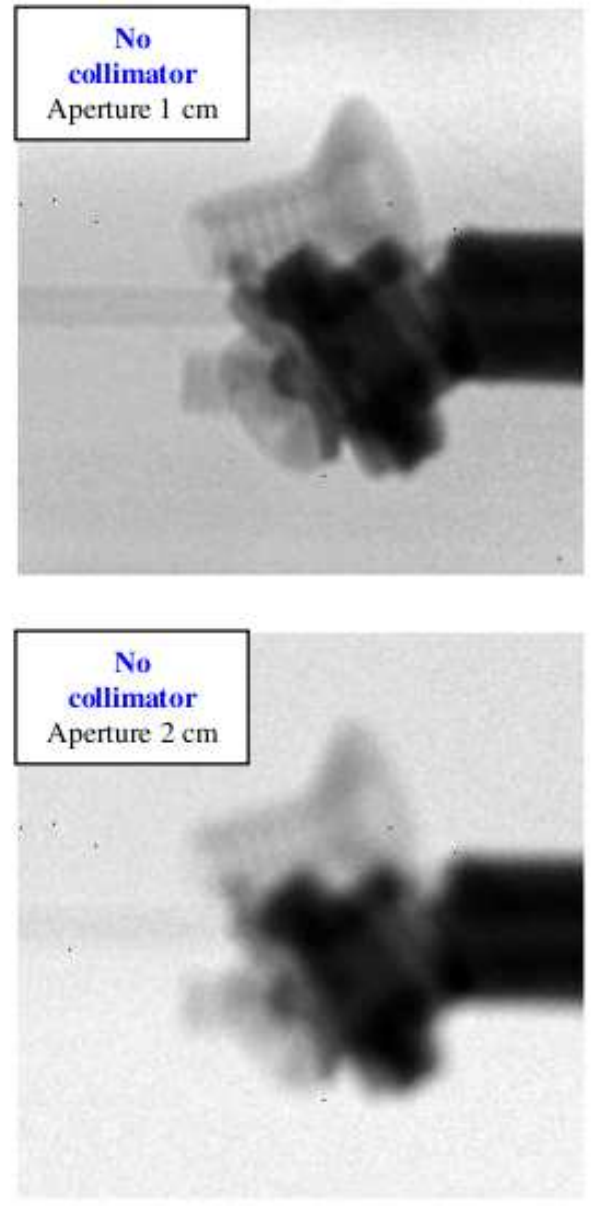

Figure 6. Neutron transmission images of different test objects, photograph of which is shown in Fig. 9. Two aperture sizes $(1 \mathrm{~cm}$ and $2 \mathrm{~cm})$ are used in the measurements $(\mathrm{L} / \mathrm{D}=116: 1$ and $\mathrm{L} / \mathrm{D}=58: 1)$. Distance between the object and detector active area is $\mathrm{L}=42 \mathrm{~mm}$. Integration time 20 and $10 \mathrm{~s}(1 \mathrm{~cm}$ and 2 $\mathrm{cm}$ aperture, respectively) for images with no collimator and 200 and $100 \mathrm{~s}$ for images obtained with the collimator installed in the beam. The improvement of spatial resolution is more pronounced at the $2 \mathrm{~cm}$ aperture (uncollimated beam L/D of 58:1).

will allow substantial improvement of spatial resolution for a fixed integration time, when full beam can be used with no apertures installed. It also indicates how important it is to achieve very efficient out-of-angle rejection.

\subsection{Neutron refraction effect}

The cold neutron spectrum of FunSpin facility leads to a presence of a strong refraction at the edges of certain metal samples. The refraction effects, which are currently studied in detail at Paul Scherrer Institute, may be helpful to improve contrast in certain radiography applications/objects and at the same time may introduce large distortions in reconstructed images, as in case of tomographic reconstructions. An example of strong refraction at the boundaries of a hypodermic needle is shown in figures 9 and 10. Our policapillary collimators, installed between the sample and the detector can be used to suppress that effect by eliminating the refracted neutrons, thus eliminating 


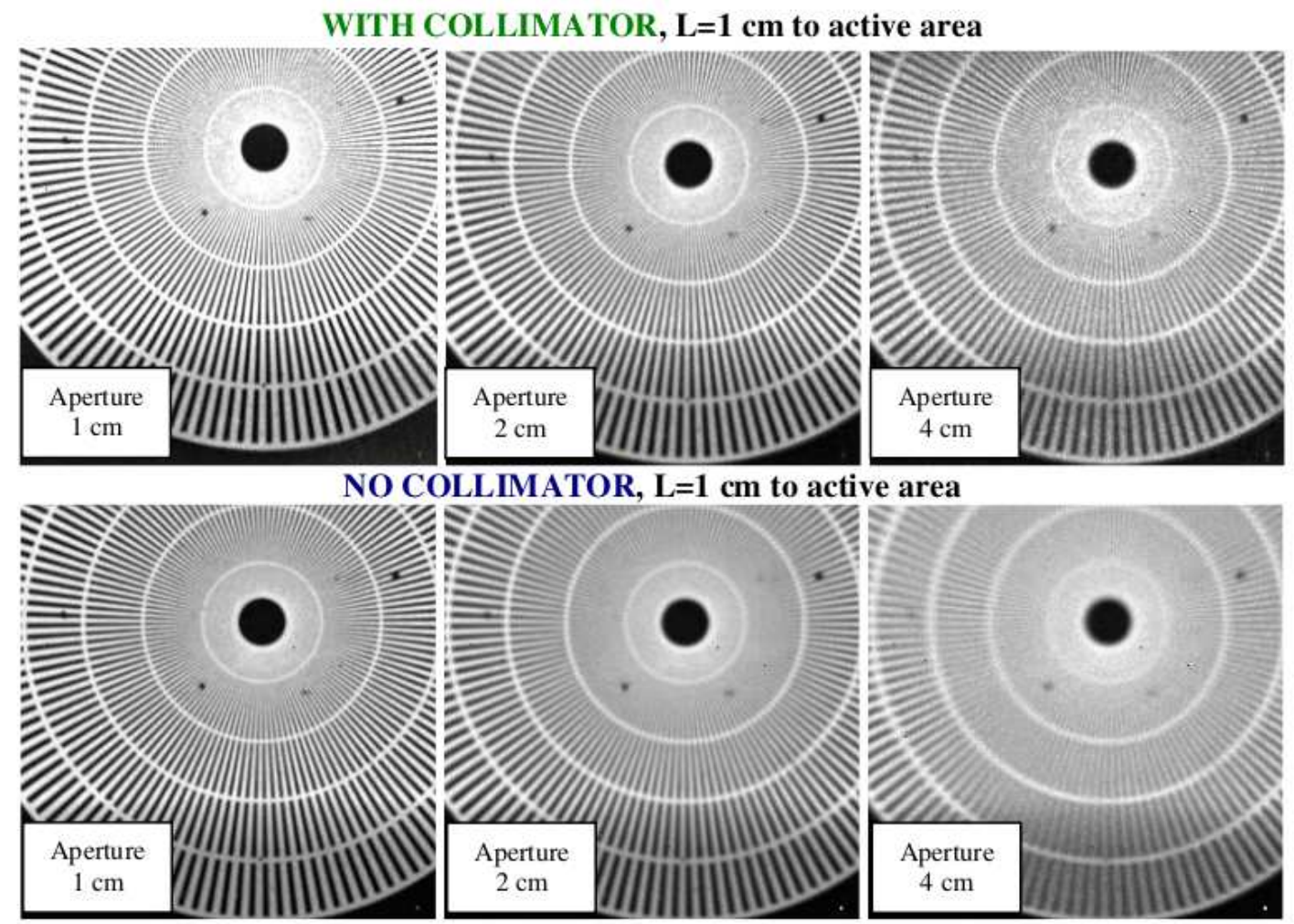

Figure 7. Neutron transmission radiographies of a PSI test pattern [15] obtained at different beam collimation defined by the aperture $(1 \mathrm{~cm}, 2 \mathrm{~cm}$, and $4 \mathrm{~cm})$. Distance between the mask and detector active area $\mathrm{L}=1 \mathrm{~cm}$. No significant improvement of spatial resolution by using a policapillary collimator is observed for the $1 \mathrm{~cm}$ aperture at this distance to the active area, while the imaging with a $4 \mathrm{~cm}$ aperture is substantially improved by the collimator. The data acquisition time for images with collimator is $300 \mathrm{~s}, 150 \mathrm{~s}$ and $30 \mathrm{~s}$ for $1 \mathrm{~cm}, 2 \mathrm{~cm}$ and $4 \mathrm{~cm}$ aperture, respectively. In case of no collimator imaging the integration time was $30 \mathrm{~s}$, $15 \mathrm{~s}$ and $3 \mathrm{~s}$, respectively.

the increased intensity at the edges $(0.9 \mathrm{~mm}$ and $2 \mathrm{~mm}$ positions at the left figure 10$)$, however collimators cannot compensate the neutron deficit at "darker" regions of the image $(1.1 \mathrm{~mm}$ and $1.7 \mathrm{~mm}$ positions of figure $10 \mathrm{left}$ ).

\section{Conclusions}

The results of our measurements at FunSpin cold neuron facility demonstrate the potential of policapillary collimators for improvement of spatial resolution in neutron imaging experiments where the samples have to be mounted at some distance from the detector or in experiments where the input aperture collimation is not sufficient due to spatial constraints of the experimental setup. In case when the samples can be mounted very close to the active area of neutron detector and the intrinsic spatial resolution of detector is not better than the beam divergence factor in that configuration no collimator should be used and image integration times can be relatively short.

The characteristics of our compact collimators have to be improved in order to make them useful for realistic applications where the image integration time is an important parameter. First 


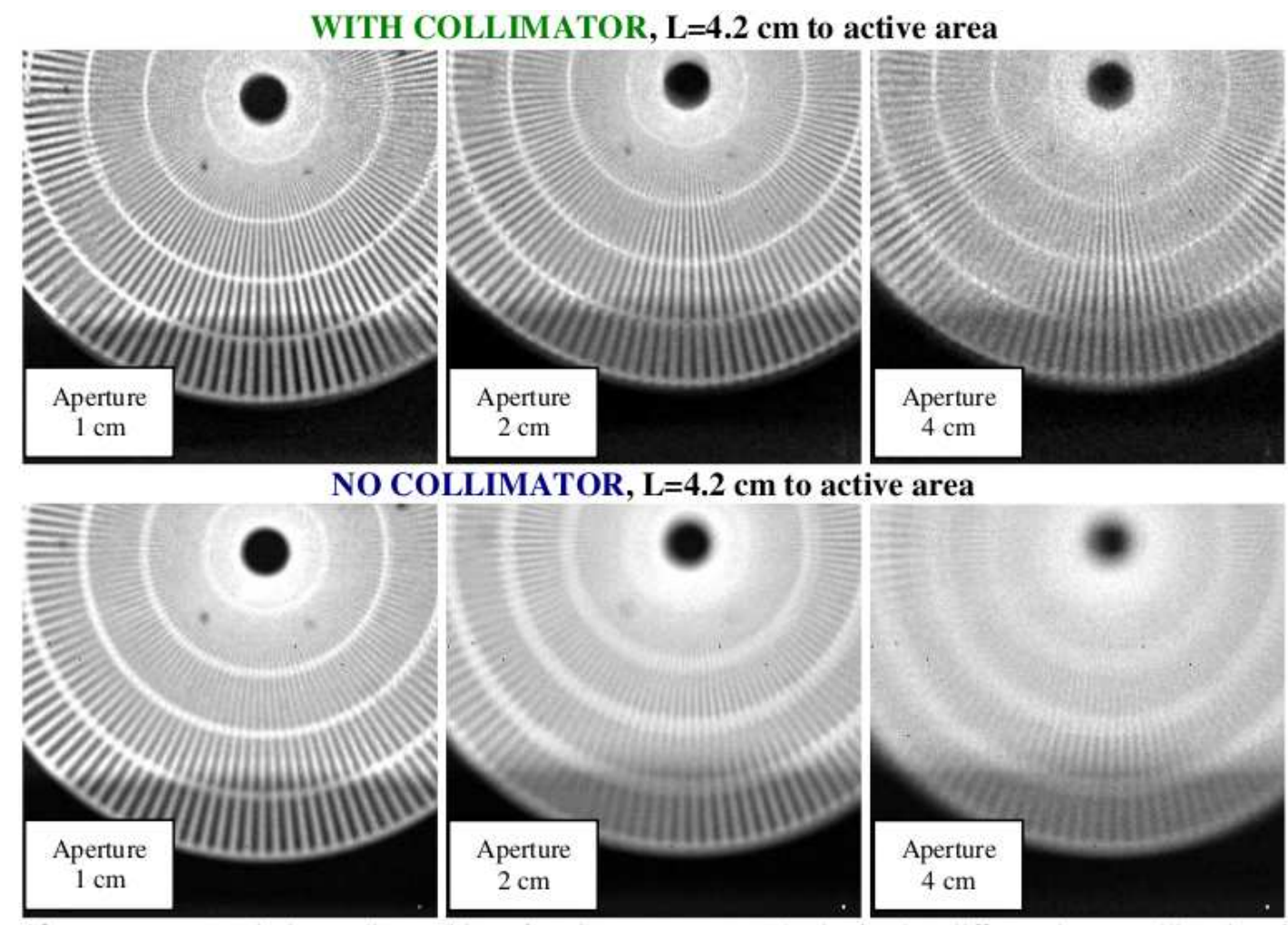

Figure 8. Neutron transmission radiographies of a PSI test pattern [15] obtained at different beam collimation defined by the aperture $(1 \mathrm{~cm}, 2 \mathrm{~cm}$, and $4 \mathrm{~cm})$. Distance between the mask and detector active area $\mathrm{L}=4.2 \mathrm{~cm}$. Significant improvement of spatial resolution by the collimator is observed for all images measured at this distance between active area and the object. Image integration times are the same as in figure 7.

of all, the improvement of the out-of-angle rejection will enable imaging with very large input apertures (ideally no apertures at all). Secondly, the increased peak transmission of collimator by an optimized device internal structure will proportionally decrease the integration time.

\section{Acknowledgments}

The authors would like to thank AllMotion of Union City, California (www.allmotion.com) for their invaluable help with control of high accuracy motorized stages required for the collimator alignment. The detector used in these experiments was developed within the Medipix collaboration. We greatly appreciate help of collaboration, in particular our colleagues at NiKHEF for the Muros readout electronics [16] and data acquisition software developed by our colleagues at the Institute of Experimental and Applied Physics, Czech Technical University in Prague [17]. This work was supported in part by the U.S. Department of Energy under STTR Grants No. DE-FG02-07ER86322 and DE-FG02-08ER86353. 

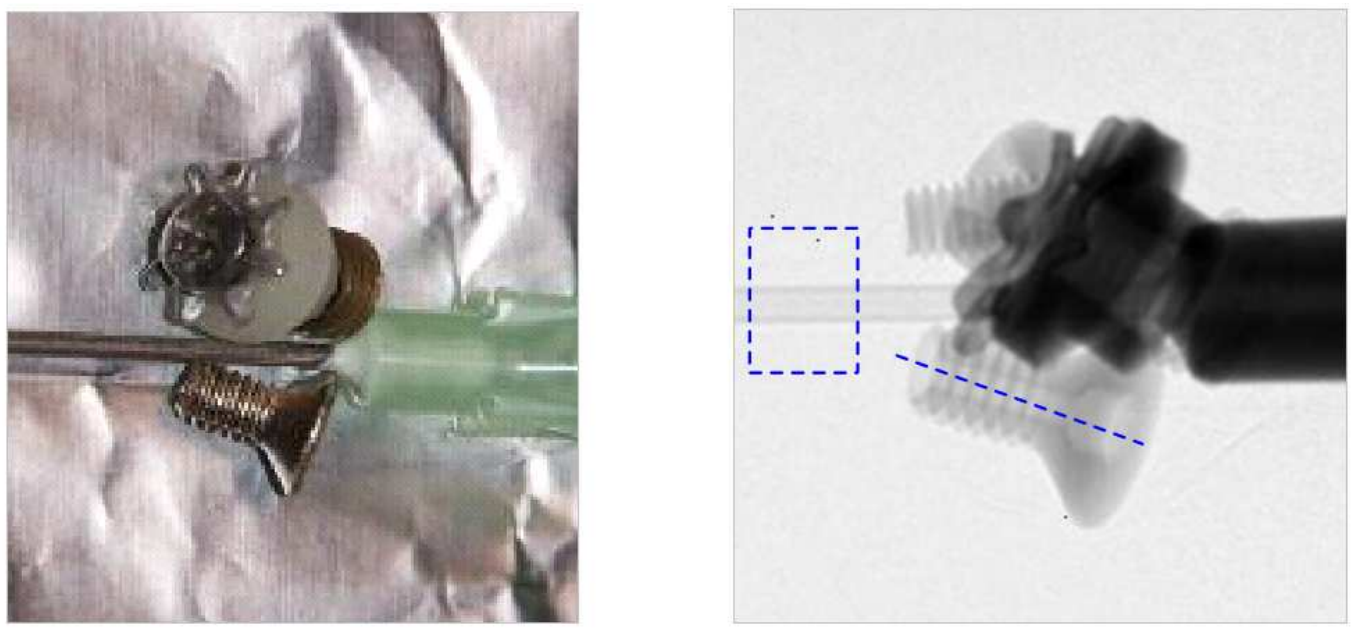

Figure 9. A photograph and neutron transmission image of a test objectconsisting of M2 steel screws and plastic washers and a hypodermic needle. Distance between the object and detector active area $\mathrm{L}=1 \mathrm{~cm}, \mathrm{no}$ MCP collimator was used, input aperture was $1 \mathrm{~cm}$. Imagge acquisition time $100 \mathrm{~s}$. The dashed line and squire indicate the areas of cross sections through the image shown in figure 10.
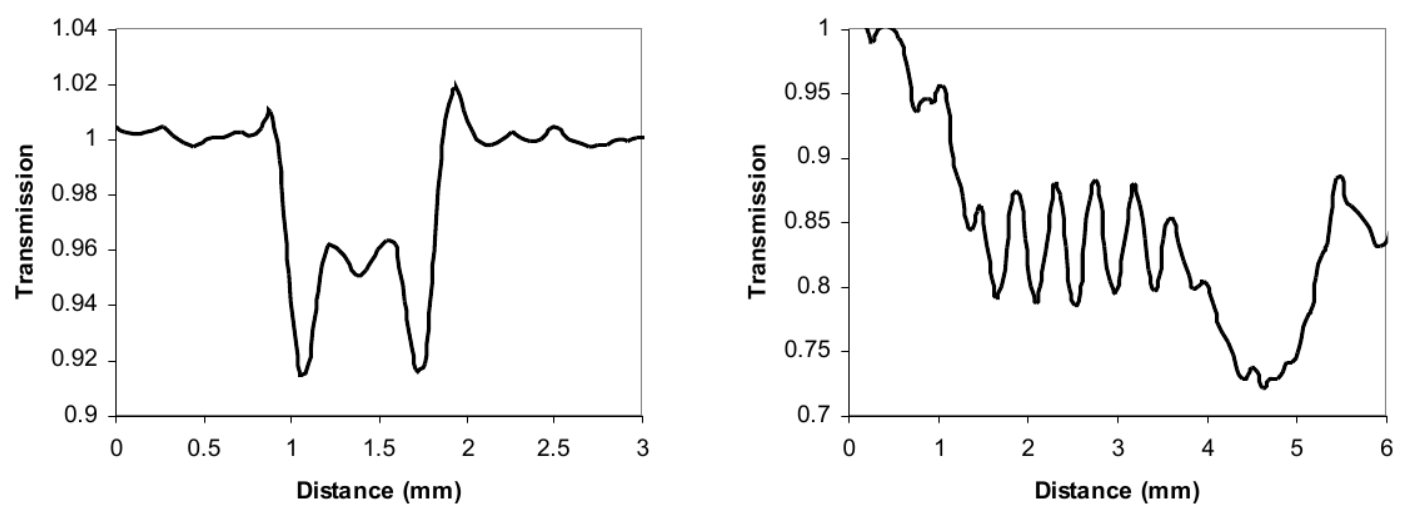

Figure 10. Cross sections through the transmission image shown in figure 9. A strong refraction effect is observed at the edges of the hypodermic needle (left). The threads of the steel screw (right) are clearly resolved at this distance to the detector with a $1 \mathrm{~cm}$ input beam aperture ( $\mathrm{L} / \mathrm{D}=116: 1$, corresponding to $\sim 86$ $\mu \mathrm{m}$ beam divergence at $\mathrm{L}=1 \mathrm{~cm}$ distance).

\section{References}

[1] E.H. Lehmann and W. Wagner, Neutron imaging at PSI: a promising tool in materials science and technology, Appl. Phys. A 99 (2010) 627.

[2] M. Strobl, I. Manke, N. Kardjilov, A. Hilger, M. Dawson and J. Banhart, Advances in neutron radiography and tomography, J. Phys. D 42 (2009) 243001.

[3] N. Kardjilov et al., Three-dimensional imaging of magnetic fields with polarized neutrons, Nature Physics 4 (2008) 399.

[4] L.D. Cussen, P. Hoghoj and I.S. Anderson, Neutron collimator with rectangular beam profile, Nucl. Instrum. Meth. A 460 (2001) 374. 
[5] F.M. Piegsa, Highly collimating neutron optical devices, Nucl. Instrum. Meth. A 603 (2009) 401.

[6] G. Frei, E.H. Lehmann, D. Mannes and P. Boillat, The neutron micro-tomography setup at PSI and its use for research purposes and engineering applications, Nucl. Instrum. Meth. A 605 (2009) 111.

[7] O.H.W. Siegmund, A high spatial resolution event counting neutron detector using microchannel plates and cross delay line readout, Nucl. Instrum. Meth. A 579 (2007) 188.

[8] A.S. Tremsin et al., On the possibility to image thermal and cold neutron with sub-15 $\mu \mathrm{m}$ spatial resolution, Nucl. Instrum. Meth. A 592 (2008) 374.

[9] A.S. Tremsin and W.B. Feller, The theory of compact and efficient circular-pore MCP neutron collimators, Nucl. Instrum. Meth. A 556 (2005) 556.

[10] A.S. Tremsin et al., Neutron Collimation with Microchannel Plates: Calibration of Existing Technology and Near Future Possibilities, IEEE Trans. Nucl. Sci. 54 (2007) 362.

[11] J. Zejma et al., FUNSPIN polarized cold-neutron beam at PSI, Nucl. Instrum. Meth. A 539 (2005) 622.

[12] J.R. Santisteban, L. Edwards, M.E. Fizpatrick, A. Steuwer and P.J. Withers, Engineering applications of Bragg-edge neutron transmission, Appl. Physica A 74 (2002) S1433.

[13] A.S. Tremsin et al., Improvement of quantitative neutron radiography by scatter rejection with policapillary collimators, proceedings of World Conf. on Neutron Radiography, to be published in Nucl. Instrum. Meth. A.

[14] A. S. Tremsin et al., High resolution neutron radiography with microchannel plates: proof-of-principle experiments at PSI, Nucl. Instrum. Meth. A 605 (2009) 103.

[15] C. Grünzweig, G. Frei, E. Lehmann, G. Kühne and C. David, Highly absorbing gadolinium test device to characterize the performance of neutron imaging detector systems, Rev. Sci. Instrum. 78 (2007) 053708.

[16] D. San Segundo Bello, M. van Beuzekom, P. Jansweijer, H. Verkooijen and J. Visschers, An interface board for the control and data acquisition of the Medipix2 chip, Nucl. Instrum. Meth. A 509 (2003) 164.

[17] T. Holy et al., Data acquisition and processing software package for Medipix2, Nucl. Instrum. Meth. A 563 (2006) 254. 